\title{
Multiparametric MRI and MR Spectroscopic Imaging of Prostate Lesions with Implementation of PI-RADS and Histopathological Correlation: A Prospective Analysis
}

\author{
Reddy Ravikanth ${ }^{1}$ Pooja Majumdar ${ }^{2}$ \\ ${ }^{1}$ Department of Radiology, Holy Family Hospital, Thodupuzha, \\ Kerala, India \\ 2Department of Medicine, INHS Sanjeevani, Willingdon Island \\ Ernakulam, Kerala, India
}

\begin{abstract}
Address for correspondence Reddy Ravikanth, MD, Department of Radiology, Holy Family Hospital, Thodupuzha 685605, Iddukki, Kerala, India (e-mail: ravikanthreddy06@gmail.com).
\end{abstract}

Asian J Oncol 2019;5:19-23

\begin{abstract}
Keywords

- prostate carcinoma

- magnetic resonance spectroscopic imaging

- PI-RADS

- prostate-specific antigen

- histopathological

Introduction Majority of the elderly male population suffers from prostatism due to enlargement of the prostate as a result of what is now regarded as male climacteric. For standardizing multiparametric magnetic resonance imaging (MRI) of the prostate, the European Society of Urogenital Radiology published Prostate Imaging Reporting and Data System (PI-RADS) in 2012. Magnetic resonance spectroscopic imaging (MRSI) uses a strong magnetic field to obtain metabolic information that identifies the relative concentrations of various metabolites in the cell cytoplasm and the extracellular space. Prostate carcinoma is associated with proportionately lower levels of citrate and higher levels of choline and creatine than are seen in benign prostatic hyperplasia (BPH) or in normal prostate. This difference can be detected by MRSI.

Aim This study has been undertaken with the objective of finding how reliable MRI is in establishing the diagnosis of lesions of prostate and to correlate the histopathological (HPE) findings of transrectal ultrasound (TRUS)-guided targeted prostate biopsy with the PI-RADS scoring (Version 2) on MRI in patients. Also, the study evaluates the accuracy of MRSI in the detection of prostatic carcinoma in men with elevated prostate-specific antigen (PSA) levels. Materials and Methods A prospective study was undertaken at a tertiary care hospital during a period of 2 years from May 2017 to April 2019 on 50 patients with PSA > 4 ng/dL and with palpable lesion on digital rectal examination. MRI and MRSI were performed with a 1.5-Tesla body MRI system. The pelvic phased-array coil was used for both excitation and signal reception. Imaging was done using standard protocols. Histological material was obtained from prostate chippings from transurethral resection of prostate. Results Fifty patients with elevated PSA levels underwent MRI, MRSI, TRUS-guided biopsy and documented PI-RADS scores. Eight patients had PI-RADS 1 score, 2 patients had PI-RADS 2 score, 8 patients had PI-RADS 3 score, 14 patients had PI-RAD 4 score, and 18 patients had PI-RADS 5 score. Thirty-two patients with PI-RADS 4 and PI-RADS 5 on imaging showed positive for malignancy on HPE, showing significant association on Chi-square test with $X^{2}=16.412$ and $p<0.001$ (statistically significant). MRSI detected 36 patients on biopsy proven cases of carcinoma (sensitivity $89.5 \%$, specificity $64 \%$, and accuracy $88 \%$ ).

Conclusion This study showed a very good correlation between positivity of prostate carcinoma on HPE and higher PI-RADS (4 and 5, respectively). PI-RADS overall score had 94\% sensitivity compared with components such as diffusion-weighted imaging (DWI, $78 \%$ ) and T2 hypointensity (72\%). MRSI is more sensitive and specific compared with T2-weighted images alone.
\end{abstract}

License terms

$($ () (1) $\ominus \circledast$ 


\section{Introduction}

In India, prostate carcinoma has an incidence rate of 3.9 per 100,000 men and is responsible for $9 \%$ of cancer-related mortality. ${ }^{1}$ Prostate carcinoma is the second most frequently diagnosed cancer and the sixth leading cause of cancer death in males worldwide; it is least common in South and East Asia, more common in Europe, and most common in the United States. According to the American Cancer Society, prostate carcinoma is the most common malignancy in American men and the second leading cause of deaths from cancer, after lung cancer. ${ }^{2}$ It is the only malignancy that is diagnosed with an apparently blind technique, that is, transrectal sextant biopsy. ${ }^{3}$ Prostate carcinoma is a common cause of morbidity and mortality in developed countries worldwide, particularly in Europe and North America. ${ }^{4}$ Prostate carcinoma differs from many other solid tumors in that the prevalence of latent disease-the number of men with undetected prostate carcinoma-far exceeds the number of men diagnosed with, or dying from, the disease. Prostatic obstruction must be diagnosed early so that a definite treatment can be instituted to relieve the patient of symptoms and to prevent the sequelae of the obstruction.

The widespread and repeated use of serum prostate-specific antigen (PSA) tests and extended-pattern transrectal ultrasound (TRUS)-guided biopsy has resulted in considerable stage migration. Few men now present with locoregional or metastatic disease identified by standard imaging (bone scan or cross-sectional pelvic imaging with magnetic resonance imaging [MRI] or computerized tomography [CT]). Therefore, most clinicians have shifted from the use of imaging-based staging paradigms to a combination of clinical variables (serum PSA, T stage, Gleason score, and extent of disease on biopsy) as a more efficient means to assess the likely extent of disease and determine the best initial treatment. ${ }^{5}$

However, despite the efficiency of clinical staging, it remains imperfect for precise local cancer staging and intraprostatic tumor localization, both of which play important roles in initial assessment, treatment, and, in many cases, follow-up. Under staging, which was reported to be in the range of $30 \%$ to cancer is often performed to exclude lymph node metastases in patients who are thought to be candidates for definitive local therapy. CT and MRI have similar sensitivities for this purpose. However, the incidence of lymph node metastases is currently low, and imaging is costly with limited sensitivity. A review of the literature that encompassed 15 series and 1,354 patients, with an incidence of lymph node metastases of $22 \%$, revealed a sensitivity of CT and MRI of $\sim 36 \%$ and a specificity of $97 \% .^{6}$

Prostate imaging anatomy as assessed by endorectal MRI utilizes a magnetic coil placed in the rectum to better visualize the zonal anatomy of the prostate and better delineate tumor location, volume, and extent (stage). Patients are imaged in a whole-body scanner, using a pelvic phased array coil combined with an inflatable, balloon-covered, endorectal surface coil positioned in the rectum. Both T1- and T2-weighted spin-echo MRI images are required to evaluate prostate carcinoma. Thin-section axial images are used for tumor localization and assessment of the extent (stage) of the tumor. Specifically, the presence of extra capsular extension (ECE) and/or seminal vesicle invasion (SVI) is noted.

Coronal imaging may be helpful for tumor localization and in assessing SVI. Prostate zonal anatomy cannot be fully appreciated on T1-weighted images, as the gland is of intermediate signal intensity on such images. However, on T2-weighted images, zonal anatomy is evident: the peripheral zone is of high-signal intensity and is surrounded by a thin rim of low-signal intensity, representing the capsule of the gland. The central and transition zones are both of lower T2 signal intensity than the peripheral zone, likely due to its smooth muscle. ${ }^{7}$ However, in the current study MRI and magnetic resonance spectroscopy imaging (MRSI) of prostate were done by pelvic phased array coil for the detection of prostatic carcinoma.

With use of three-dimensional (3D) MRSI, significantly higher choline and significantly lower citrate levels were observed in regions of cancer compared in those areas of benign hypertrophy and normal prostate tissue. With increasing numbers of high-Tesla MRI equipment being installed in India, the radiologist needs to be cognizant about endorectal MRI and multiparametric imaging for prostate carcinoma. ${ }^{8}$ Advanced prostate carcinoma may present with bone pain from metastases, anemia, and renal failure, due to obstructive changes or, rarely, spinal cord compression. Early prostate carcinoma, however, is mainly asymptomatic with its detection often being the result of an elevated PSA test. PSA was first described in 1979 and later proposed as a marker for prostate carcinoma. ${ }^{9}$ It is a kallikrein-like serine protease and is produced almost exclusively by the epithelial cells of the prostate. ${ }^{10}$

Grading follows the Gleason system established in $1974 .{ }^{11}$ The Gleason grade is achieved by looking at the histological architecture of the prostate. The two most commonly seen patterns are added together to give the patient a final Gleason score. A final score will therefore be between 2 and 10 , with 2 being the least and 10 the most aggressive type of cancer. Histological material is obtained from prostate chippings from a patient undergoing a transurethral resection of prostate. The standard approach to prostatic biopsy is to biopsy each sextant of the prostate and in addition to sampling the peripheral zone at apex laterally and the base. ${ }^{12}$ Biopsy cores obtained in this way include the peripheral zone which is the most common location for early prostate carcinoma.

\section{Aim}

This study has been undertaken with the objective of finding how reliable MRI is in establishing the diagnosis of lesions of prostate and to correlate the histopathological (HPE) findings of TRUS-guided targeted prostate biopsy with the PI-RADS scoring (Version 2) on MRI in patients. The study also evaluated the accuracy of MRSI in the detection of prostatic carcinoma in men with elevated PSA levels.

\section{Materials and Methods}

A prospective study was undertaken at a tertiary care hospital during a period of 2 years from May 2017 to April 2019 on 50 patients diagnosed with prostatic carcinoma with PSA $>4 \mathrm{ng} / \mathrm{dL}$ and with palpable lesion on digital rectal examination. Patients 
with history of prostatic biopsy in past 6 weeks were excluded from the study. After obtaining informed written consent, 50 patients who were suspected for prostatic carcinoma were subjected to multiparametric MRI, followed by ultrasonography-guided 12 core biopsy. HPE result obtained was correlated with PI-RADS score. MRI and MRSI were undertaken using phase array body coil on GE SIGNA (General Electric Medical Systems, Milwaukee, Wisconsin, USA) 1.5 Tesla.

\section{TRUS-Guided 12-Core Biopsy}

Before the procedure, the patients were given broad-spectrum antibiotics to protect them against infection. They were also given rectal enema to empty the rectal canal before the procedure to obtain clear images. Intrarectal instillation of $20 \mathrm{~mL}$ of local anesthetic gel (lidocaine $2 \%$ ) was used to alleviate pain and discomfort during the procedure. A transrectal ultrasound probe (6-12 MHz range) with a combination of end-viewing and side-viewing transducer attached to GE LOGIQ P5 (General Electric Medical Systems, Milwaukee, Wisconsin, USA) ultrasound machine was used. Local anesthetic gel (lidocaine $2 \%$ ) was applied over a latex condom applied onto the probe. A full urinary bladder was ensured to help in better visualization of the gland prior to the procedure. All patients were examined and biopsied in the left lateral decubitus position and it was well tolerated. The prostate was imaged in both axial and sagittal planes with assessment of volume, echogenicity, surface, calcification, and the presence of nodules. Each nodule was assessed for size, location in the gland, morphology, echogenicity, margin, and extent. MRI of prostate finalized were analyzed by a radiologist with 2-year experience and PI-RADS score were documented. HPE result was collected within 7 days to minimize error and was correlated with PI-RADS score.

\section{Statistical Analysis}

The measures of association between quantitative data was done for categorical variables and assessed using chi-square test. Analysis was done using IBM SPSS Statistics (IBM Corp., Armonk, New York, USA) for Windows Version 20.0. The inference is considered statistically significant whenever $p \leq 0.05$. HPE findings were considered as the standard of reference and sensitivity, specificity, positive predictive value, negative predictive value, and accuracy were calculated for PI-RADS, MRSI, diffusion-weighted imaging (DWI), and T2 intensity.

\section{Results}

Fifty patients with elevated PSA levels underwent MRI, MRSI, TRUS-guided targeted biopsy, and documented PI-RADS

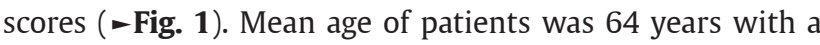
range of 52-79 years. Eight patients had PI-RADS 1 score, 2 patients had PI-RADS 2 score, 8 patients had PI-RADS 3 score, 14 patients had PI-RADS 4 score, and 18 patients had PI-RADS 5 score. Thirty-two patients with PI-RADS 4 and PI-RADS 5 on imaging showed positive for malignancy on HPE showing significant association on Chi-square test with $\chi^{2}=16.412$ and $p<0.001$ (statistically significant) (-Table $\mathbf{1}$ ). Out of 50 patients who underwent MRI, 45 patients showed T2 hypointensity and 32 patients were positive on HPE. Forty patients showed diffusion positivity and 34 patients were positive on HPE which was statistically significant with $p$-value of 0.010 . Sensitivity of diagnosing malignant lesions on PI-RADS was $94 \%$, specificity $100 \%$, positive predictive value $100 \%$, negative predictive value $98.6 \%$, and accuracy $86 \%$ (-Table 2). With results of HPE as reference standard, DWI detected 34 patients proven to have cancer on

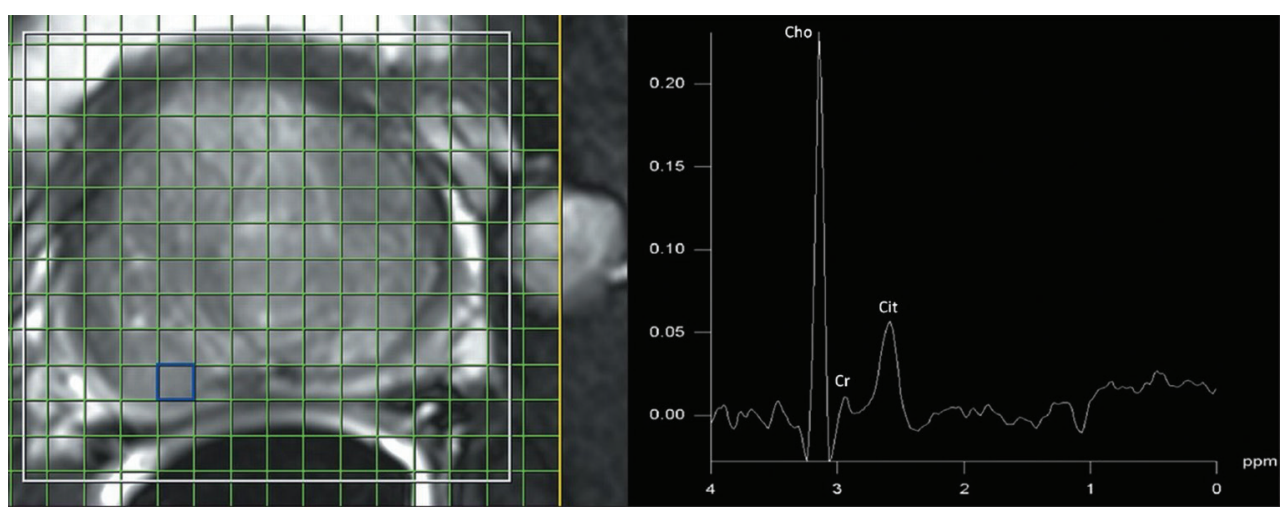

Fig. 1 MRSI spectral grid superimposed on a T2-weighted (fat-sat) image of the prostate. MR spectrum obtained from the voxel shows elevated choline and decreased citrate suggestive of malignancy. MRSI, Magnetic resonance spectroscopic imaging.

Table 1 Comparison between the PI-RADS scoring of prostate carcinoma and HPE results

\begin{tabular}{|c|c|c|c|c|c|}
\hline PI-RADS score & $\begin{array}{l}\text { Number of positive } \\
\text { cases on imaging }\end{array}$ & $\begin{array}{l}\text { Number of HPE } \\
\text { malignant cases }\end{array}$ & $\begin{array}{l}\text { Number of biopsy } \\
\text { negative cases }\end{array}$ & Chi-square test & $p$-Value \\
\hline 1 & 8 & - & - & \multirow{5}{*}{16.412} & \multirow{5}{*}{$<0.001$} \\
\hline 2 & 2 & - & - & & \\
\hline 3 & 10 & 4 & 6 & & \\
\hline 4 & 12 & 10 & 2 & & \\
\hline 5 & 18 & 17 & 1 & & \\
\hline
\end{tabular}

Abbreviations: HPE, histopathological; PI-RADS, Prostate Imaging Reporting and Data System. 
biopsy (sensitivity 78\%, specificity $43.4 \%$, and accuracy $74 \%$ ). T2-weighted images detected 32 patients proven to have cancer on biopsy (sensitivity $72 \%$, specificity $41.6 \%$, and accuracy $72 \%$ ). MRSI detected 36 patients proven to have cancer on biopsy (sensitivity $89.5 \%$, specificity $64 \%$, accuracy $88 \%)$. MRSI was more sensitive and specific compared with T2-weighted images alone (- Table 3; - Fig. 2A-C).

\section{Discussion}

This study showed a very good correlation with positivity of prostate carcinoma on HPE with higher PI-RADS (4 and 5). The study conducted by Kuru et $\mathrm{al}^{13}$ and five other studies observed positive correlation with prostate carcinoma detection and higher PI-RADS score and reduced incidence of prostate carcinoma with overall PI-RADS $<3$ when correlated with HPE. As a result, it can be inferred that multiparametric MRI with PI-RADS scoring reduces the need for biopsy in men with PI-RADS $<3$, while at the same time improving the overall rate of detection of intermediate/high-risk prostate carcinoma. Pokorny et al $^{14}$ also observed that multiparametric MRI reduces need of biopsy in patients with lower overall PI-RADS score. Overall, PI-RADS scoring in the current study showed $94 \%$ sensitivity compared with that of its individual components alone (DWI, 78\%, T2 hypointensity 72\%), which was similar to observation done by Junker et al. ${ }^{15}$

MRSI enables noninvasive assessment of certain metabolites in the prostate gland. Several studies ${ }^{16}$ have demonstrated that this metabolic information, in combination with anatomical information from T2-weighted MRI significantly improves prostate carcinoma detection, localization, and disease characterization. The technology of MRSI is continuously evolving with improvements of hardware and acquisition methods. MRSI has been demonstrated to be valuable in the diagnosis, localization, and characterization of the disease. It was included in the original PI-RADS, but not in the most recent version, PI-RADS version 2.0, due to the low practicality of current MRSI methods in routine clinical use.

Table 2 Comparison between PI-RADS, T2-weighted MRI, and MRSI

\begin{tabular}{|l|l|l|l|}
\hline & PI-RADS score & T2-weighted MRI & MRSI \\
\hline True positive & 36 & 32 & 35 \\
\hline True negative & 11 & 5 & 6 \\
\hline False positive & 2 & 7 & 4 \\
\hline False negative & 1 & 6 & 5 \\
\hline Total & 50 & 50 & 50 \\
\hline Sensitivity & $94 \%$ & $72 \%$ & $89.5 \%$ \\
\hline Specificity & $100 \%$ & $41.6 \%$ & $64.0 \%$ \\
\hline Positive predictive Value & $100 \%$ & $82.0 \%$ & $91.2 \%$ \\
\hline Negative predictive Value & $98.6 \%$ & $45.2 \%$ & $58.5 \%$ \\
\hline Accuracy & $86.0 \%$ & $72.0 \%$ & $88.0 \%$ \\
\hline
\end{tabular}

Abbreviations: MRI, magnetic resonance imaging; MRSI, Magnetic resonance spectroscopic imaging; PI-RADS, Prostate Imaging Reporting and Data System.

Table 3 Comparison between HPE, PI-RADS, T2-weighted MRI, and MRSI

\begin{tabular}{|c|c|c|c|}
\hline \multirow[t]{3}{*}{ Positivity on imaging } & \multicolumn{2}{|c|}{ HPE } & \multirow[t]{2}{*}{ Total } \\
\hline & Positive & Negative & \\
\hline & \multicolumn{2}{|c|}{ PI-RADS } & \\
\hline Positive & 36 & 6 & 42 \\
\hline Negative & 0 & 8 & 8 \\
\hline Total & 36 & 14 & 50 \\
\hline \multicolumn{4}{|l|}{ DWI } \\
\hline Positive & 34 & 6 & 40 \\
\hline Negative & 1 & 9 & 10 \\
\hline Total & 35 & 15 & 50 \\
\hline \multicolumn{4}{|l|}{ T2 signal intensity } \\
\hline Hypointense & 32 & 13 & 45 \\
\hline Hyperintense & 1 & 4 & 5 \\
\hline Total & 33 & 17 & 50 \\
\hline
\end{tabular}

Abbreviations: DWI, diffusion-weighted imaging; MRI, magnetic resonance imaging; MRSI, Magnetic resonance spectroscopic imaging; PI-RADS, Prostate Imaging Reporting and Data System. 

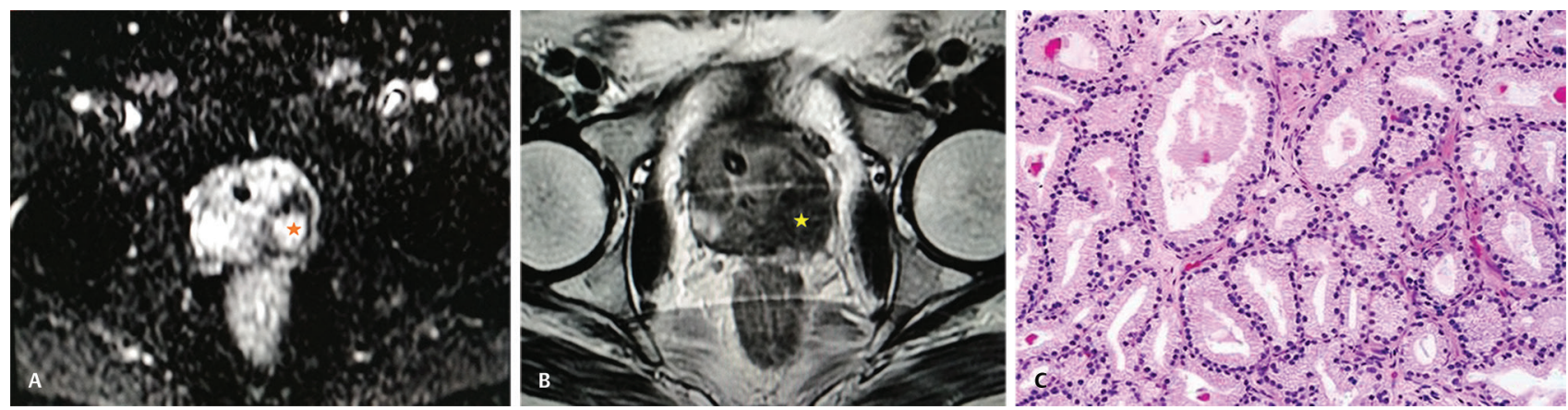

Fig. 2 (A) High signal intensity on axial diffusion-weighted MR image indicating restricted diffusion (asterisk). (B) Axial T2-weighted MR image demonstrating low signal intensity lesion (asterisk) in the peripheral zone indicative of malignancy. (C) Histopathology proven case of prostate adenocarcinoma demonstrating uniform acini which are well demarcated from the intervening stroma (H\&E, x20). MR, magnetic resonance; H\&E, haemotoxylin and eosin.

\section{Conclusion}

Multiparametric MRI of prostate has great utility in early detection of prostate carcinoma. PI-RADS version 2 is an improvement of the older version and it is a reliable tool for better communication between radiologists and clinicians in patients evaluated for prostate carcinoma. In addition to that, PI-RADS version 2 scoring has significantly improved decision making with regard to need and guidance for biopsy. The current study demonstrated that MRI and MRSI of the prostate have high accuracy in detection of organ confined prostate carcinoma and differentiating it from benign hyperplasia. MRSI is more sensitive and specific than MRI alone and combined imaging with MRI and MRSI is recommended. Demonstration of cancer to the confines of prostatic capsule is one of the most important roles of imaging in prostatic carcinoma and determination of a management strategy. MRI has significant accuracy in detection of organ confined disease.

\section{Limitations}

Small sample size is a limitation and also PI-RADS scoring was done by a single radiologist. Multiple radiologists reviewing the same could have better scoring of PI-RADS.

\section{Funding \\ None.}

\section{Conflict of Interest}

None declared.

\section{References}

1 Hedgire SS, Oei TN, McDermott S, Cao K, Patel M Z, Harisinghani MG. Multiparametric magnetic resonance imaging of prostate cancer. Indian J Radiol Imaging 2012;22(3):160-169

2 Mettlin C. The American Cancer Society National Prostate Cancer Detection Project and National patterns of prostate cancer detection and treatment. CA Cancer J Clin 1997;47(5):265-272

3 Gupta NP, Ansari MS, Dass SC. Transrectal ultrasound guided biopsy for detecting early prostate cancer: an Indian experience. Indian J. Cancer 2005;42(3):151-154

4 Merrill RM, Feuer EJ, Warren JL, Schussler N, Stephenson RA. Role of transurethral resection of the prostate in population-based prostate cancer incidence rates. Am J Epidemiol 1999;150(8):848-860
5 Richie JP, Catalona WJ, Ahmann FR, et al. Effect of patient age on early detection of prostate cancer with serum prostate-specific antigen and digital rectal examination. Urology 1993;42(4):365-374

6 Wang MC, Valenzuela LA, Murphy GP, Chu TM. Purification of a human prostate specific antigen. Invest Urol 1979;17(2):159-163

7 Fütterer JJ, Barentsz JO, Heijmink SW. Value of 3-T magnetic resonance imaging in local staging of prostate cancer. Top Magn Reson Imaging 2008;19(6):285-289

8 Cruz M, Tsuda K, Narumi Y, et al. Characterization of low-intensity lesions in the peripheral zone of prostate on pre-biopsy endorectal coil MR imaging. Eur Radiol 2002;12(2):357-365

9 Foster LS, Jajodia P, Fournier G, Jr. Shinohara K, Carroll P, Narayan $P$. The value of prostate specific antigen and transrectal ultrasound guided biopsy in detecting prostatic fossa recurrences following radical prostatectomy.JUrol 1993;149(5):1024-1028

10 Stamey TA, Yang N, Hay AR, McNeal JE, Freiha FS, Redwine E. Prostate-specific antigen as a serum marker for adenocarcinoma of the prostate. N Engl J Med 1987;317(15): 909-916

11 Gleason DF, Mellinger GT. Prediction of prognosis for prostatic adenocarcinoma by combined histological grading and clinical staging. J Urol 1974;111(1):58-64

12 Aus G, Bergdahl S, Hugosson J, Lodding P, Pihl CG, Pileblad E. Outcome of laterally directed sextant biopsies of the prostate in screened males aged 50-66 years. Implications for sampling order. Eur Urol 2001;39(6):655-660

13 Kuru TH, Roethke MC, Rieker P, et al. Histology core-specific evaluation of the European Society of Urogenital Radiology (ESUR) standardised scoring system of multiparametric magnetic resonance imaging (mpMRI) of the prostate. BJU Int 2013;112(8):1080-1087

14 Pokorny MR, de Rooij M, Duncan E, et al. Prospective study of diagnostic accuracy comparing prostate cancer detection by transrectal ultrasound-guided biopsy versus magnetic resonance (MR) imaging with subsequent MR-guided biopsy in men without previous prostate biopsies. Eur Urol 2014;66(1):22-29

15 Junker D, Schäfer $G$, Edlinger $M$, et al. Evaluation of the PI-RADS scoring system for classifying mpMRI findings in men with suspicion of prostate cancer. BioMed Res Int 2013;2013(1):252939

16 Kurhanewicz J, Swanson MG, Nelson SJ, Vigneron DB. Combined magnetic resonance imaging and spectroscopic imaging approach to molecular imaging of prostate cancer. J Magn Reson Imaging 2002;16(4):451-463 